\title{
MicroScale Thermophoresis (MST) for studying actin polymerization kinetics
}

\author{
Andrea Topf, Peter Franz, and Georgios Tsiavaliaris \\ Institute for Biophysical Chemistry, Hannover Medical School, \\ Hannover, Germany
}

BioTechniques 63:187-190 (October 2017) doi 10.2144/000114599

Keywords: microscale thermophoresis; actin, polymerization; actin-binding protein; kinetics

Here, we present a MicroScale Thermophoresis (MST)-based assay for in vitro assessment of actin polymerization. By monitoring the thermophoretic behavior of ATTO ${ }^{488}$-labeled actin in a temperature gradient over time, we could follow polymerization in real time and resolve its three characteristic phases: nucleation, elongation, and steady-state equilibration. Titration experiments allowed us to evaluate the effects of actin-binding proteins (ABPs) on polymerization, including DNase I-induced inhibition and mDia2FH1FH2 (mDia2)-assisted acceleration of nucleation. The corresponding rates of actin filament elongation were quantitatively determined, yielding values in good agreement with those obtained using the pyrene-actin polymerization assay. Finally, we measured the effect of myosin on actin polymerization, circumventing the problems of fluorescence quenching and signal disturbance that occur with other techniques. MST is a simple and valuable research tool for investigating actin kinetics covering a wide range of molecular interactions, with low protein consumption.

Detection and measurement of actin polymerization are essential for understanding actin cytoskeleton dynamics and the influential role of actin-binding proteins (ABPs) in actin regulation. Light scattering (1), viscometry (2), and the use of fluorophores (3) are classic methods used to study in vitro the process by which actin monomers (G-actin) assemble into larger filaments (F-actin) (Figure 1A). Although these techniques can determine parameters such as critical concentration, filament elongation rate, and rate of disassembly, they have limitations. The signal in scattering experiments depends not only on polymer concentration but also on filament size and filament-bound proteins. Filament fragmentation limits the accuracy of viscometry measurements, and fluorimetric approaches such as the pyreneactin polymerization assay (3) often face the problem of fluorescence quenching (4). Although alternative techniques based on total internal reflection microscopy (TIR-FM) have recently grown in importance for studying actin dynamics (5), the requirement for specialized instrumentation and laborious data analysis make TIR-FM time-consuming, costly, and difficult for high-throughput analysis.

Here, we developed a new assay based on MicroScale Thermophoresis (MST) to monitor actin polymerization in real time using low-microliter sample volumes. MST makes use of thermophoresis-directed movement of molecules in a temperature gradient according to their size, charge, and hydration shell (6). Since molecular interactions affect at least one of these parameters, the molecules' thermophoretic movements change accordingly and can be tracked by MST, providing a quantitative measure of the equilibrium reaction. Fluorescence labeling is only required for detection and can be omitted if the probe displays intrinsic fluorescence properties (7). For these reasons, MST has emerged as a highly sensitive technique for studying molecular interactions under steady-state conditions $(8,9)$.

The standard MST device used in this study (Monolith NT.115; NanoTemper Technologies, Munich, Germany) is operated by the NTControl v2.1.33 software and is composed of up to 16 glass capillaries, a set of light-emitting diodes (LEDs) and detectors for fluorescence-based tracking of molecule movements, and an infrared (IR)-laser system (Figure 1B) that creates a local $2-6^{\circ} \mathrm{C}$ temperature gradient to induce thermophoresis (10). We carried out actin polymerization using a mixture of G-actin and ATTO ${ }^{488}-\mathrm{G}$-actin. Briefly, G-actin was labeled with a 2-fold molar excess of ATTO $^{488}$-maleimide (28562 SIGMA; Sigma-Aldrich Chemie $\mathrm{GmbH}$, Munich, Germany) in $2 \mathrm{mM}$ Tris- $\mathrm{HCl}$ buffer (Merck, Darmstadt, Germany), pH 8.0, 0.2 $\mathrm{mM} \mathrm{CaCl}_{2}, 0.1 \mathrm{mM}$ ATP (SigmaAldrich, Munich, Germany) under gentle stirring for $5 \mathrm{~h}$ in the dark at $4^{\circ} \mathrm{C}$ and then purified by size-exclusion chromatography (Superdex S75 26/600 column; GE Healthcare, Freiburg, Germany). Concentration and labeling efficiency were determined photometrically using the extinction coefficients $\varepsilon_{290 \mathrm{~nm}}\left(\mathrm{G}\right.$-actin): $26600 \mathrm{M}^{-1} \mathrm{~cm}^{-1}$; $\varepsilon_{501 \mathrm{~nm}}\left(\mathrm{ATTO}^{488}\right.$-maleimide): $90000 \mathrm{M}^{-1} \mathrm{~cm}^{-1}$; and $\varepsilon_{290 \mathrm{~nm}}\left(\mathrm{ATTO}^{488}\right.$-maleimide): 9450 $\mathrm{M}^{-1} \mathrm{Cm}^{-1}$. IR-laser power was set to $40 \%$, and the blue LED channel with 40\% power was used for fluorescence excitation. Experiments were performed using 40 nM ATTO ${ }^{488}$-G-actin and varying amounts of unlabeled G-actin and ABPs.

\section{METHOD SUMMARY}

MicroScale Thermophoresis (MST) is a simple, fast, and cost-effective tool for studying actin polymerization kinetics in real time. The method is based on quantification of thermophoresis- and temperature-induced changes (T-jump) in fluorescence intensity of ATTO ${ }^{488}$-labeled actin. 

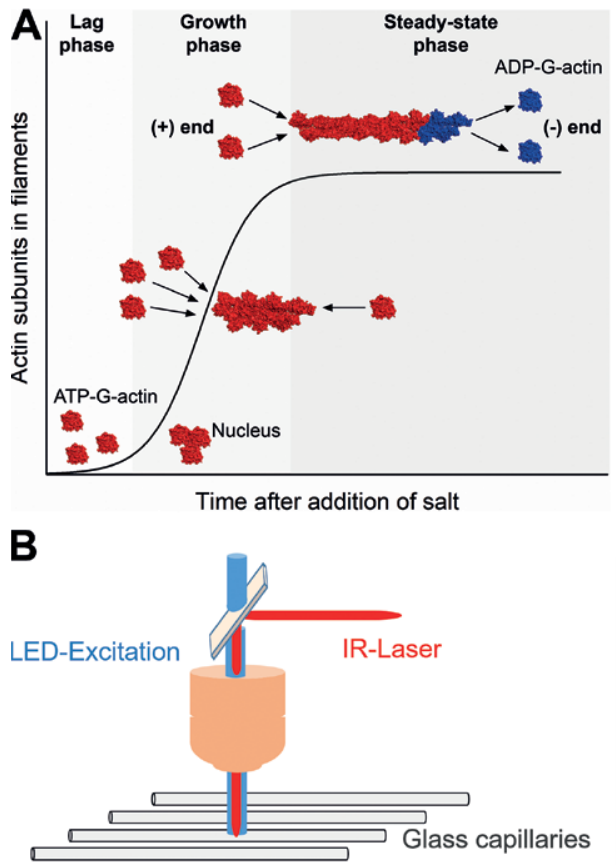
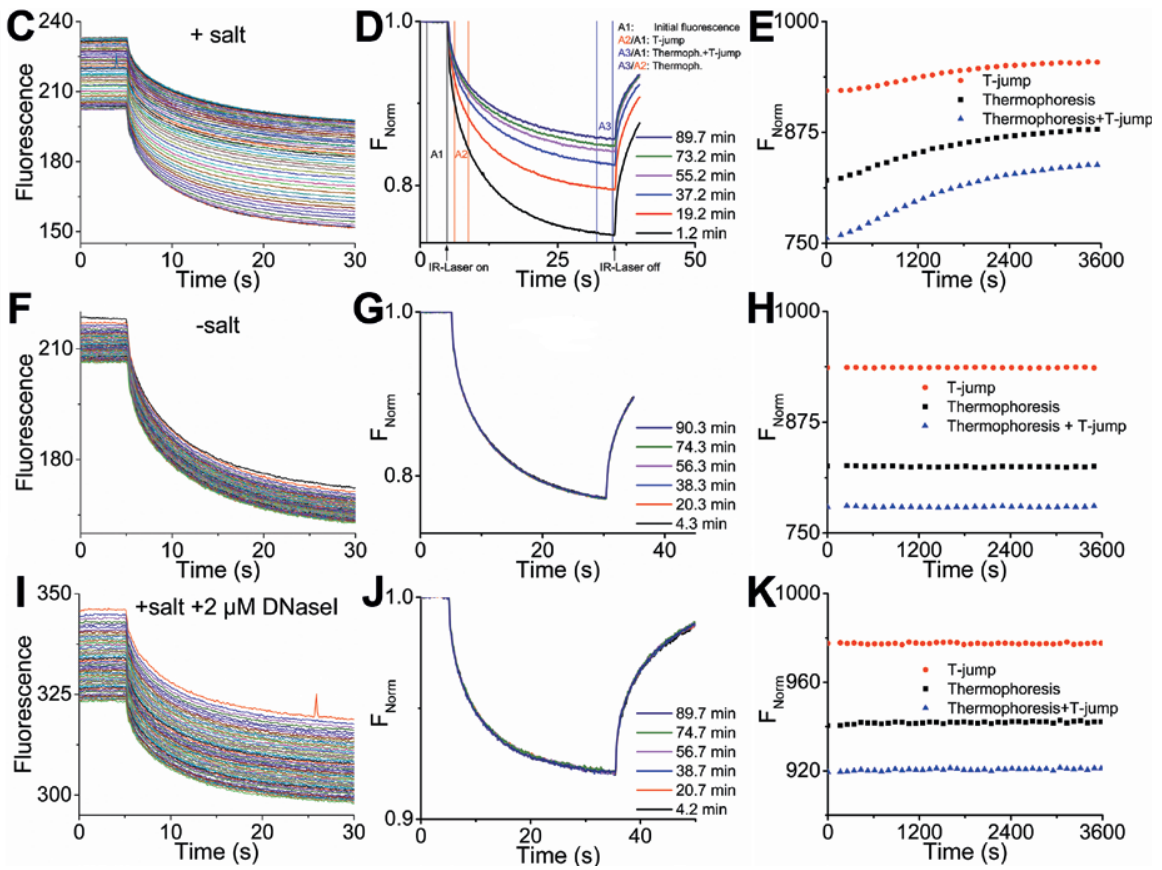

Figure 1. MicroScale Thermophoresis (MST)-based actin polymerization assay. (A) Illustration of the three sequential steps of actin polymerization. During the lag phase, ATP-G-actin monomers assemble and form small nuclei composed of three to four monomers. The growth phase describes the fast exponential kinetics of nuclei elongation and actin filament formation. Actin subunits are incorporated at both ends, with the barbed (+)-end elongating considerably faster than the pointed (-)-end. During filament growth, ATP is hydrolysed, and phosphate is released, resulting in stable ADP-F-actin. At steady-state, filaments maintain a constant length over time due to balanced kinetics of ATP-G-actin association with the (+)-end and ADP-G-actin dissociation from the (-)-end, a process known as treadmilling. Actin binding proteins (ABPs) and other factors can influence polymerization. (B) Graphical representation of the MST setup. (C) Raw fluorescence traces of an actin polymerization reaction with time. (D) Normalized MST fluorescence traces after initiation of polymerization at defined time points. Margins mark fluorescence before (A1), after induction of a temperature gradient (A2) corresponding to temperature jump (T-jump), and at thermophoretic equilibrium (A3). Depletion of the thermophoretic profiles with time (colored lines) reflects reaction progress. (E) Plots of normalized fluorescence differences over polymerization time as obtained from thermophoresis (ratio A3:A2, black squares), T-jump (ratio A2:A1, red circles), and the combination of T-jump and thermophoresis (ratio A3:A1, blue triangles). (F) Raw fluorescence traces of actin in low-salt buffer over time. (G) Fluorescence profiles of $2 \mu \mathrm{M}$ G-actin under non polymerizing (- salt) conditions and $(\mathrm{H})$ corresponding thermophoresis, T-jump, and thermophoresis plus T-jump data. (I) Raw fluorescence traces of actin in the presence of equimolar DNase I concentration with time. $(J)$ Normalized fluorescence profiles in the presence of DNase I and (K) corresponding thermophoresis, T-jump, and thermophoresis plus T-jump data. Stable fluorescence profiles indicate effective suppression of polymerization. All graphs were prepared using Origin 2016G software (OriginLab, Northampton, MA).

Figure $1 \mathrm{C}$ shows representative fluorescence traces of a polymerization experiment over 90 min performed in a single capillary (MO-K002 Monolith, NanoTemper Technologies) filled with $6 \mu \mathrm{L}$ actin solution $\left[40 \mathrm{nM}\right.$ ATTO ${ }^{488}-\mathrm{G}-$ actin/1.96 $\mu \mathrm{M}$ G-actin in Tris-HCl buffer supplemented with $0.5 \mathrm{mM}$ DTT, (SigmaAldrich)]. Concentrations of ATTO ${ }^{488}$ labeled actin $>40 \mathrm{nM}$ in the sample solution had a slight inhibitory effect on polymerization. Polymerization was initiated by addition of $2 \mathrm{mM} \mathrm{MgCl}$, and $100 \mathrm{mM} \mathrm{KCl}$ (Sigma-Aldrich) before the sample was aspirated into the capillary tubes. Figure 1D shows a selection of normalized recordings obtained from raw fluorescence using the NT Analysis 1.5.41 software. A single trace comprises three time intervals: before, during, and after induction of a temperature gradient. Colored traces reflect fluorescence

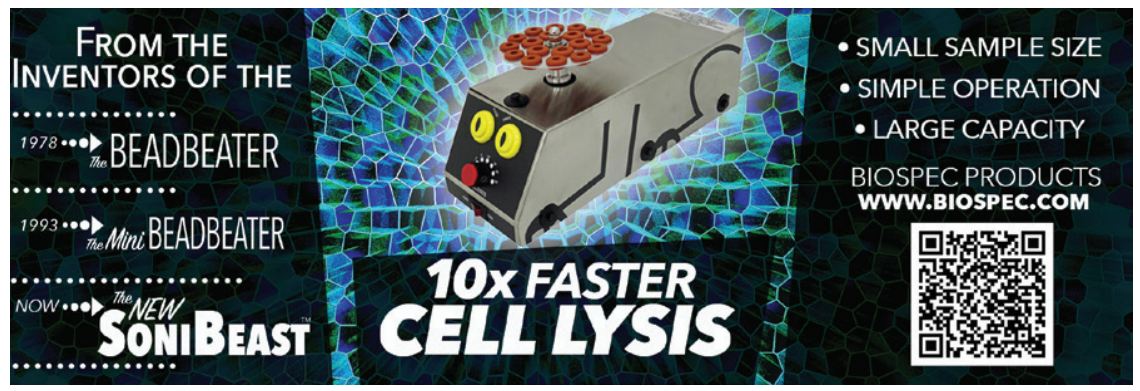

changes over time corresponding to changes in thermophoresis and temperature-induced changes in fluorescence intensity [temperature jump (T-jump)]. The steep decrease in fluorescence reflects positive thermophoresis as molecules move toward regions of lower temperature until equilibrium is reached, followed by an increase in fluorescence due to back-diffusion of the molecules. Plotting time-dependent changes in fluorescence as ratios of intensities at the defined time intervals (10), which correspond to changes in thermophoresis (ratio A3:A1), T-jump (ratio A2:A1), and both thermophoresis and T-jump (ratio A3:A2), we obtained sigmoidal curves characteristic for actin polymerization that reached a plateau after 3000 s (Figure 1E).

To verify that the observed timedependent change in MST signal amplitude indeed reflects the kinetics of actin polymerization, we repeated the 
Table 1. Summary of elongation rates of actin polymerization.

\begin{tabular}{|c|c|c|c|c|c|c|c|c|}
\hline \multirow[t]{2}{*}{$\begin{array}{c}\text { G-actin } \\
\text { concentration }\end{array}$} & \multicolumn{2}{|c|}{ Thermophoresis } & \multicolumn{2}{|c|}{ T-jump } & \multicolumn{2}{|c|}{$\begin{array}{l}\text { Thermophoresis } \\
\text { + T-jump }\end{array}$} & \multicolumn{2}{|c|}{$\begin{array}{c}\text { Pyrene-actin } \\
\text { polymerization assay }\end{array}$} \\
\hline & \multicolumn{8}{|c|}{$k\left(10^{-3} s^{-1}\right)$} \\
\hline $0.5 \mu \mathrm{M}$ & 2.0 & n.a. & 1.5 & n.a. & 1.6 & n.a. & 1.8 & n.a. \\
\hline $2 \mu \mathrm{M}^{*}$ & 11 & $40 \pm 1$ & 10 & $46 \pm 1$ & 8.7 & $36 \pm 0.5$ & 11 & $32 \pm 1$ \\
\hline+1 nM mDia2 $2^{\#}$ & \multicolumn{2}{|c|}{$119 \pm 12$} & \multicolumn{2}{|c|}{$84 \pm 5$} & \multicolumn{2}{|c|}{$124 \pm 8$} & \multicolumn{2}{|c|}{$85 \pm 12$} \\
\hline + $5 \mathrm{nM}$ mDia2 & \multicolumn{2}{|c|}{$433 \pm 24$} & \multicolumn{2}{|c|}{$340 \pm 21$} & \multicolumn{2}{|c|}{$408 \pm 27$} & \multicolumn{2}{|c|}{$345 \pm 14$} \\
\hline + $1 \mathrm{nM}$ myosin* & 9 & $40 \pm 1$ & 11 & $47 \pm 2$ & 24 & $46 \pm 3$ & & \\
\hline$+1 \mu \mathrm{M}$ myosin & \multicolumn{2}{|c|}{$463 \pm 6$} & \multicolumn{2}{|c|}{$480 \pm 156$} & \multicolumn{2}{|c|}{$465 \pm 58$} & \multicolumn{2}{|c|}{ n.a. } \\
\hline $5 \mu \mathrm{M}^{\#}$ & \multicolumn{2}{|c|}{$222 \pm 15$} & \multicolumn{2}{|c|}{$150 \pm 8$} & \multicolumn{2}{|c|}{$135 \pm 17$} & \multicolumn{2}{|c|}{$120 \pm 9$} \\
\hline \multicolumn{9}{|c|}{$\begin{array}{l}\text { Parameters in the left boxes show the elongation rate within the lag phase, as obtained from the slope between } \\
\text { the origin and the earliest time point at approximately } 75 \mathrm{~s} \text {. All other parameters display the maximum elon- } \\
\text { gation rates of the exponential growth phase obtained from the slope at the inflection point. Errors represent } \\
\text { standard error of linear fitting around the inflection point. *Under these conditions, both the lag phase (left) and } \\
\text { the exponential growth phase (right) of actin polymerization were visible. }{ }^{*} \text { A lag phase is missing under these } \\
\text { conditions. T-jump: temperature jump; mDia2: mDia2FH1FH2. }\end{array}$} \\
\hline
\end{tabular}

experiments using conditions that abolish polymerization, either by omitting salt or by adding the actin capper DNase I (grade Il from bovine pancreas; Sigma-Aldrich) to the solution. The latter prevents polymerization by tightly binding to the barbed ends of actin monomers (11). In both cases, fluorescence profiles remained constant over time (Figure 1, F-K), indicating a complete inhibition of polymerization. The sensitivity of the method toward changes in polymer concentration was further evaluated with different initial G-actin concentrations (Figure 2A, left three panels). Elongation rates (Table 1) were similar to those obtained with the pyrene-actin polymerization assay (Figure $2 \mathrm{~A}$, right panel) and in good agreement with previous findings (12). At concentrations less than the critical concentration [0.5 $\mathrm{MM}$ mDia2FH1FH2 (mDia2)], a long lag phase, rate-limited by the nucleation process, determined the kinetics. This lag phase disappeared at higher G-actin concentrations, while elongation rates increased according to pseudo-first order kinetics (3).

To test whether MST can also be used to study polymerization under control of ABPs, we performed experiments with the actin nucleator mDia2 (Figure 2B). The elongation rates (Table 1) reflected those obtained with the pyrene-actin polymerization assay (Figure 2B, right panel), revealing the potency of mDia2 to accelerate polymerization through nucleation activity as previously described (13). Even the overshoot of actin polymerization, defined as a peak in actin polymer concentration followed by a decrease due to fragmentation or hydrolysis, as often observed for ABPs that accelerate polymerization, becomes visible in the thermophoresis data. These experiments reaffirm that MST is a highly sensitive analytical tool for measuring changes in polymer concentration and support previous assertions that the overshoot is not an artifact (14). Moreover, using our assay, we determined the rate by which myosin accelerates actin polymerization (Figure 2C; Table 1), a process that cannot be easily resolved using the pyrene-actin polymerization assay and is difficult to quantitatively assess by lightscattering approaches (4).

Our data demonstrate the potential of using MST to address kinetic aspects of protein assembly. As exemplified by monitoring the actin polymerization process using microliter sample volumes and low fluorophore concentrations with the option to run up to 16 experiments in parallel, MST reduces protein consumption and shortens experimental time while providing high sensitivity. According to our and the manufacturer's experience, temporal resolution can be further improved by monitoring thermophoretic motions for only a few ature gradient and analysis of the T-jump associated changes in fluorescence. Thus, polymerization reactions occurring on the tens of seconds to minutes time scale can efficiently be monitored by our MST-based approach, which makes the method particularly useful for cytoskeletal research to resolve mechanistic details behind the complex nature of the actin polymerization reaction and its regulation, including its potential use to screen for seconds after initiation of the temper-

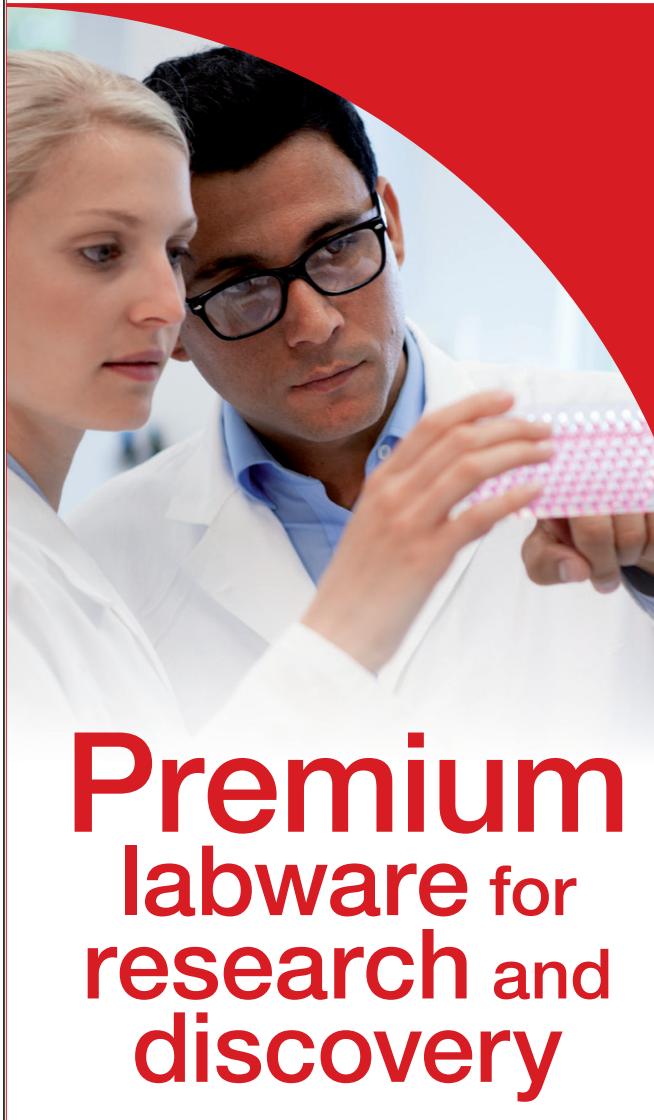

\section{Cell and Tissue Culture}

- Three different color coded growth surfaces

- Optimized, user-friendly geometries

- Labeling of all products with LOT number and expiration date

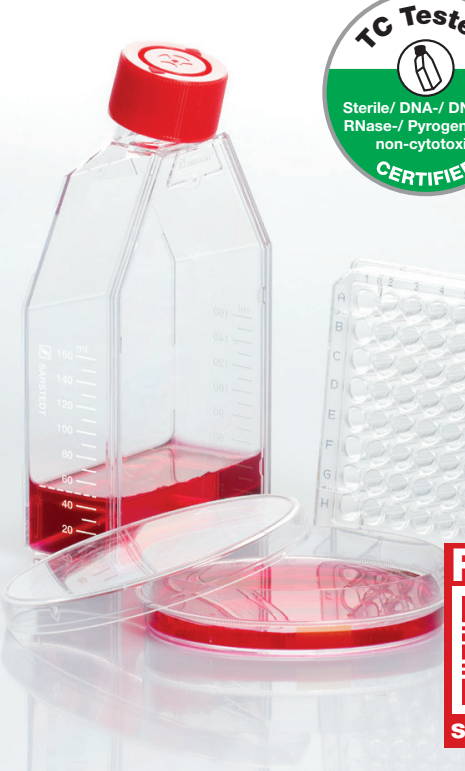

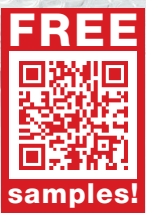



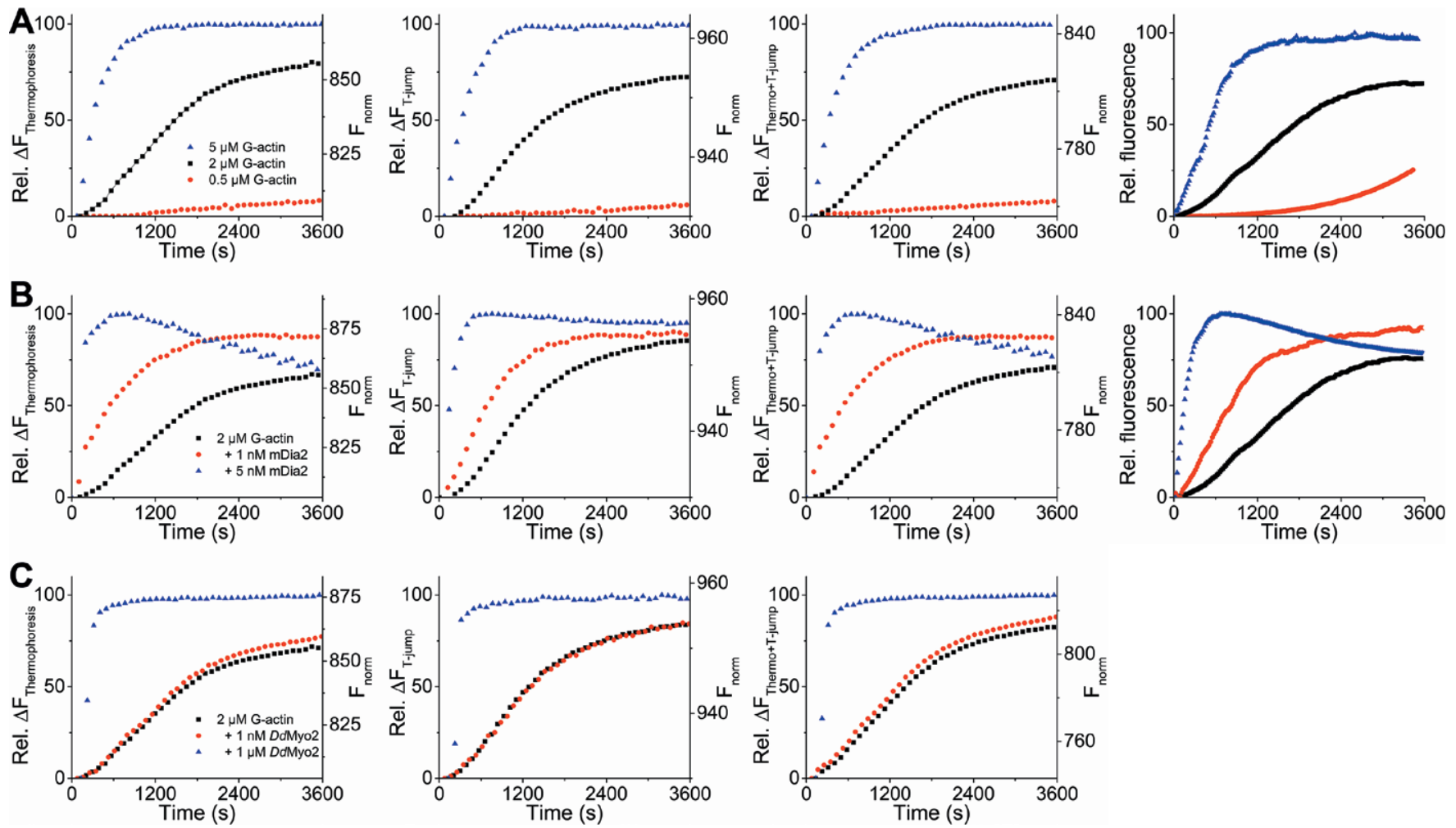

Figure 2. Comparison of the MicroScale Thermophoresis (MST)-based actin polymerization assay with the classical pyrene-actin polymerization assay. (A) Time course of actin polymerization obtained by MST (Panels 1-3) and with the pyrene-actin polymerization assay (right panel). Concentrations of G-actin used were: $0.5 \mu \mathrm{M}, 2 \mu \mathrm{M}$, and $5 \mu \mathrm{M}$. (B) Actin polymerization in the absence and presence of mDia2FH1FH2 (mDia2). Time course of actin polymerization obtained MST (left 3 panels) and pyrene-actin fluorescence data (right panel). (C) Accelerated actin polymerization kinetics in the presence of micromolar concentrations of a recombinant myosin-2 single head fragment referred to as DdMyo2 (15). All experiments were performed at high salt conditions. Data points are averages from two to three independent experiments. Left and right y-axes show normalized and non-normalized thermophoresis and temperature jump (T-jump) data, respectively.

chemical compounds that target the cytoskeleton and associated proteins (e.g., inhibitors of actin, ABPs, or tubulin) for cell biological and therapeutic applications.

\section{Author contributions}

A.T. and P.F. prepared protein and performed experiments. A.T., P.F., and G.T. analyzed the data. G.T. conceived and supervised the study and wrote the paper.

\section{Acknowledgments}

We thank Christian Waßmann for technical assistance, Jan Faix (Hannover Medical School) for providing mDiaFH1FH2, and NanoTemper Technologies for discussions.

\section{Competing interests}

The authors declare no competing interests.

\section{References}

1. Masai, J., S. Ishiwata, and S. Fujime. 1986. Dynamic light-scattering study on polymerization process of muscle actin. Biophys. Chem. 25:253-269.
2. MacLean-Fletcher, S.D. and T.D. Pollard. 1980. Viscometric analysis of the gelation of Acanthamoeba extracts and purification of two gelation factors. J. Cell Biol. 85:414-428.

3. Kouyama, T. and K. Mihashi. 1981. Fluorimetry study of $\mathrm{N}$-(1-pyrenyl)iodoacetamide-labelled F-actin. Local structural change of actin protomer both on polymerization and on binding of heavy meromyosin. Eur. J. Biochem. 114:33-38.

4. Miller, L., M. Phillips, and E. Reisler. 1988. Polymerization of $\mathrm{G}$-actin by myosin subfragment 1. J. Biol. Chem. 263:1996-2002.

5. Amann, K.J. and T.D. Pollard. 2001. Direct real-time observation of actin filament branching mediated by Arp2/3 complex using total internal reflection fluorescence microscopy. Proc. Natl. Acad. Sci. USA 98:15009-15013.

6. Duhr, S. and D. Braun. 2006. Why molecules move along a temperature gradient. Proc. Natl. Acad. Sci. USA 103:19678-19682.

7. Seidel, S.A., C.J. Wienken, S. Geissler, M. Jerabek-Willemsen, S. Duhr, A. Reiter, D. Trauner, D. Braun, and P. Baaske. 2012. Labelfree microscale thermophoresis discriminates sites and affinity of protein-ligand binding. Angew. Chem. Int. Ed. Engl. 51:10656-10659.

8. Taft, M.H., E. Behrmann, L.C. MunskeWeidemann, C. Thiel, S. Raunser, and D.J. Manstein. 2013. Functional characterization of human myosin-18A and its interaction with F-actin and GOLPH3. J. Biol. Chem. 288:30029-30041.

9. Nienberg, C., C. Garmann, A. Gratz, A. Bollacke, C. Gotz, and J. Jose. 2017. Identification of a Potent Allosteric Inhibitor of Human Protein Kinase
CK2 by Bacterial Surface Display Library Screening. Pharmaceuticals (Basel) 10. 10.3390/ph10010006.

10. Jerabek-Willemsen, M., C.J. Wienken, D. Braun, P. Baaske, and S. Duhr. 2011. Molecular interaction studies using microscale thermophoresis. Assay Drug Dev. Technol. 9:342-353.

11. Kabsch, W., H.G. Mannherz, D. Suck, E.F. Pai, and K.C. Holmes. 1990. Atomic structure of the actin:DNase I complex. Nature 347:37-44.

12. Pollard, T.D. 1983. Measurement of rate constants for actin filament elongation in solution. Anal. Biochem. 134:406-412.

13. Block, J., T.E. Stradal, J. Hanisch, R. Geffers, S.A. Kostler, E. Urban, J.V. Small, K. Rottner, and J. Faix. 2008. Filopodia formation induced by active mDia2/Drf3. J. Microsc. 231:506-517.

14. Brooks, F.J. and A.E. Carlsson. 2008. Actin polymerization overshoots and ATP hydrolysis as assayed by pyrene fluorescence. Biophys. J. 95:1050-1062

15. Fujita-Becker, S., G. Tsiavaliaris, R. Ohkura, T. Shimada, D.J. Manstein, and K. Sutoh. 2006. Functional characterization of the $\mathrm{N}$-terminal region of myosin-2. J. Biol. Chem. 281:36102-36109.

Received 29 June 2017; accepted 07 August 2017.

Address correspondence to Georgios Tsiavaliaris, Institute for Biophysical Chemistry, Hannover Medical School, Carl-Neuberg-Str. 1, 30625 Hannover, Germany. E-mail: tsiavaliaris.georgios@mh-hannover.de

To purchase reprints of this article, contact: biotechniques@fosterprinting.com 